\title{
Compact Multifringe Interferometry with Subpicometer Precision
}

\author{
Katharina-Sophie Isleif," Gerhard Heinzel, Moritz Mehmet, and Oliver Gerberding ${ }^{\dagger}$ \\ Max Planck Institute for Gravitational Physics (Albert Einstein Institute), Callinstrasse 38, 30167 Hannover, \\ Germany and Leibniz Universität Hannover, Institut für Gravitationsphysik, Callinstrasse 38, \\ 30167 Hannover, Germany
}

(Received 12 March 2019; revised manuscript received 6 August 2019; published 13 September 2019)

\begin{abstract}
Deep-frequency-modulation interferometry combines optical minimalism with multifringe readout. However, precision is key for applications such as optical gradiometers for satellite geodesy or as dimensional sensors for ground-based gravity experiments. We present a single-component interferometer smaller than a cubic inch. Two of these are compared to each other to demonstrate tilt and displacement measurements with a precision of less than $20 \mathrm{nrad} / \sqrt{\mathrm{Hz}}$ and $1 \mathrm{pm} / \sqrt{\mathrm{Hz}}$ at frequencies below $1 \mathrm{~Hz}$.
\end{abstract}

DOI: 10.1103/PhysRevApplied.12.034025

\section{INTRODUCTION}

Heterodyne laser interferometry, as used very successfully in the Laser Interferometer Space Antenna (LISA) Pathfinder mission [1], provides subpicometer test-mass displacement and subnanoradian tilt-sensing sensitivities, at readout frequencies between $1 \mathrm{~Hz}$ and $1 \mathrm{mHz}$, while operating over a large dynamic range (multiple fringes). Many applications and experiments would benefit from the incorporation of multiple sensors with this precision, such as future satellite-geodesy missions with multiple test masses [2], accelerometers [3], and gravitational-wave detectors [4-6]. Further applications can also make use of this precision to measure any other derived quantity at low readout frequencies, either as a core part of larger instruments or as auxiliary sensors to monitor, for example, thermal expansion or parasitic motions, without requiring subfringe stability of the objects under test. Currently, though, the availability and usability of such interferometric sensors is limited by their optical complexity. The need for intricate design, the construction of ultrastable optical benches with multiple components - many of which need individual alignment and bonding - and multiple fiber connections makes their application very elaborate. These factors drive the instrument size, mass, construction effort, and lead time and limit the current range of deployment significantly.

\footnotetext{
*katharina-sophie.isleif@web.de

$\dagger$ contact@olivergerberding.com
}

Published by the American Physical Society under the terms of the Creative Commons Attribution 4.0 International license. Further distribution of this work must maintain attribution to the author(s) and the published article's title, journal citation, and DOI.
Hence, alternative interferometer techniques that require simpler optical setups with fewer optical components [7] have the potential to reach a wider scope. Self-homodyning interferometers with phase-shift keying methods, such as digitally enhanced interferometry [8] and deep-phasemodulation $[9,10]$ or deep-frequency-modulation interferometry (DFMI) [11-13], are attractive techniques that reduce the optical complexity at the expense of more sophisticated phase-extraction algorithms. In recent years, many proof-of-principle studies [8,13-18] have revealed greatly simplified optical readout schemes that rely on such hybrid approaches. Effectively, they combine continuouswave laser sources with specific modulations to create pulselike or comblike fields that enable optical simplification, phase readout, and sometimes even multiplexing. However, probing and achieving the low-frequency displacement noise - required, for example, for future gravity experiments - has remained rare for these techniques [18].

We focus on studying DFMI, a technique that has recently gained wide interest [19-22]. Compared with the classic heterodyne scheme or homodyne quadrature interferometry [3], DFMI requires fewer optical components, which allows for more compact layouts without giving up the multifringe capabilities. Previous studies have verified this functionality [13] and here we present an experiment that demonstrates the actual displacement-sensing performance of DFMI on the $1 \mathrm{pm} / \sqrt{\mathrm{Hz}}$ level, making it an attractive alternative to heterodyne interferometry even for the most sensitive gravity experiments.

\section{EXPERIMENT}

DFMI uses a single laser beam that is strongly modulated sinusoidally in its frequency. By injecting this light into an interferometer with unequal arms, as shown in Fig. 1, we generate an output pattern that contains complex 


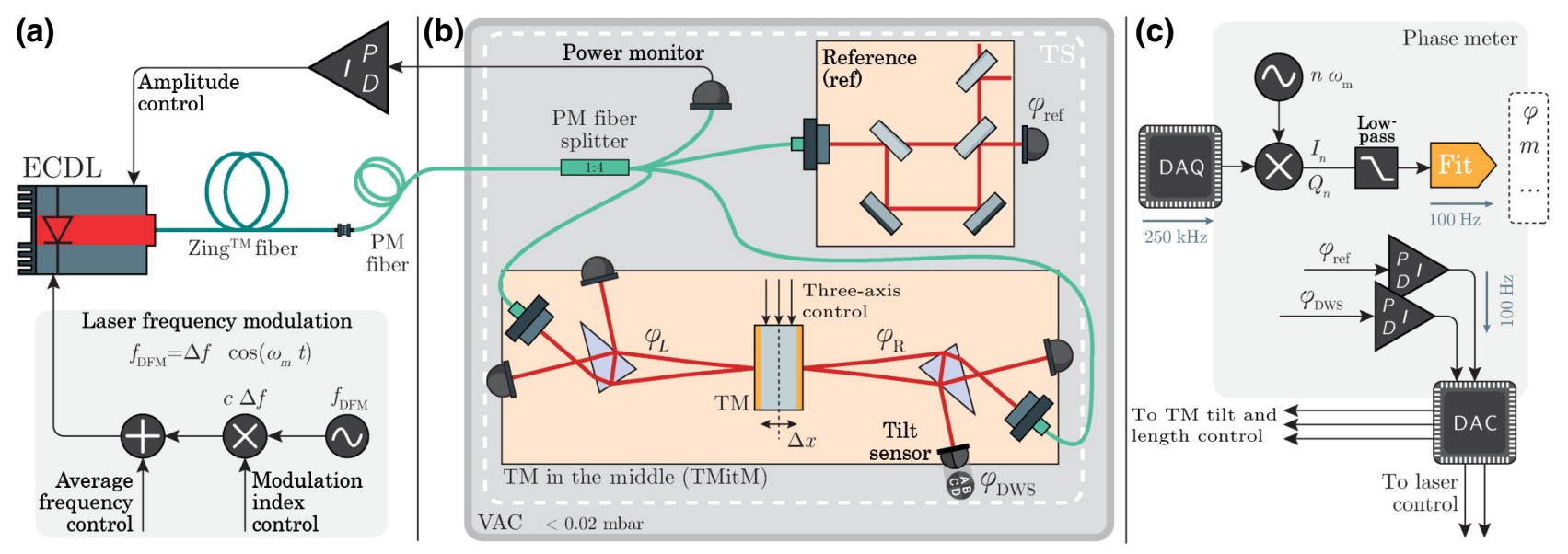

FIG. 1. A sketch of the experiment. Inset (a) shows the laser preparation. A deep frequency modulation, $f_{\mathrm{DFM}}$, is applied to a fibercoupled external-cavity diode laser (ECDL). All fiber components are polarization maintaining (PM). The vacuum chamber (VAC), shown in inset (b), houses a thermal shielding (TS) that covers two quasimonolithic interferometers: the reference (ref) and the test mass in the middle (TMitM). The data-acquisition (DAQ) system has eight analog inputs that are simultaneously digitized and processed in a software phase meter, both shown in inset (c). Analog control voltages are provided by a digital-to-analog converter (DAC) and are used to actuate on the laser and the test mass (TM).

amplitudes at the modulation frequency and its harmonics. The essence of DFMI, as we use it, is the phase-extraction algorithm: We employ a nonlinear-fit algorithm based on a Levenberg-Marquardt (least-squares) routine. It uses a Bessel function decomposition of the complex amplitudes at the modulation frequency and its first - about ten - harmonics [10]. This allows us to extract four fundamental measurement parameters in real time: the interferometric phase $\varphi$ and amplitude, the modulation index $m$, and the modulation phase, as shown in Fig. 1(c). The effective modulation index depends on the FM deviation, $\Delta f$, and on the interferometer arm-length mismatch, $\Delta L$. It is given by the relation $m=2 \pi \Delta f \Delta L / c_{0}$, with the speed of light $c_{0}$. By increasing the FM deviation, the size of the optical setups can be reduced. Estimates of absolute distances are also possible with this readout scheme by tracking the modulation index $m$ [11].

\section{A. Laser}

As illustrated in Fig. 1(a), the laser source is a fibercoupled external-cavity diode laser (ECDL) with a center wavelength of $\lambda=1064 \mathrm{~nm}$ that is modulated by $\Delta f \approx$ $\pm 5 \mathrm{GHz}$ with a rate of $f_{m}=0.8 \mathrm{kHz}$. It provides $15 \mathrm{~mW}$ optical power, which is routed into a vacuum chamber [shown in inset (b)]. A polarization-maintaining fiber splitter distributes the light equally inside the chamber via four ports to a power monitor, the so-called TMitM experiment, and to another quasimonolithic interferometer that is used as frequency reference. This optical reference allows us to measure the FM deviation and the laser frequency noise accumulated over a $7-\mathrm{cm}$ arm-length mismatch and to stabilize both [23]. Amplitude fluctuations, caused by the strong frequency-modulation and polarization fluctuations in the fibers, are actively stabilized by a closed-loop control of the laser diode current with $100 \mathrm{kHz}$ unity gain frequency.

\section{B. Test mass in the middle}

The TMitM is the key part of this experiment to test DFMI. A photograph of the interferometers is shown in Fig. 2. It consists of a 4-mm-thick mirror that is gold coated on both sides and mounted on a three-axis piezotransducer (PZT) that is glued in the center of an optical bench (OB). The OB is made of the glass ceramic Clearceram, which has a low coefficient of thermal expansion of $1 \times 10^{-8} / \mathrm{K}$. Two interferometers, one on each side of the TM, allow us to perform two redundant interferometric measurements of the same TM motion.

For this, a single-component prism-shaped interferometer is designed by using the optical simulation tool IFOCAD [24]. It has a triangular base surface with two equal sides of about $25 \mathrm{~mm}$ in length. The dimensions are chosen such that commercially available prisms made of fused silica can be used. Only the perpendicularity between the optical surfaces and base is critical. It must be better than $2^{\prime \prime}$ to ensure a sufficient vertical alignment of a monolithic interferometer. The internal prism angles are compensated during alignment by prism rotation and input beam tilts, requiring no stringent production tolerances or excessive alignment procedures. Tolerable TM rotations before loss of heterodyne efficiency are also comparable to classic interferometers with the same beam parameters. In our case, this corresponds to a heterodyne efficiency of more than $30 \%$ for $\pm 250 \mu \mathrm{rad}$ tip and tilt angles and $\pm 2 \mathrm{~mm}$ TM displacement. A sketch of the component with laser propagation and coatings is shown in the inset of Fig. 2. The 


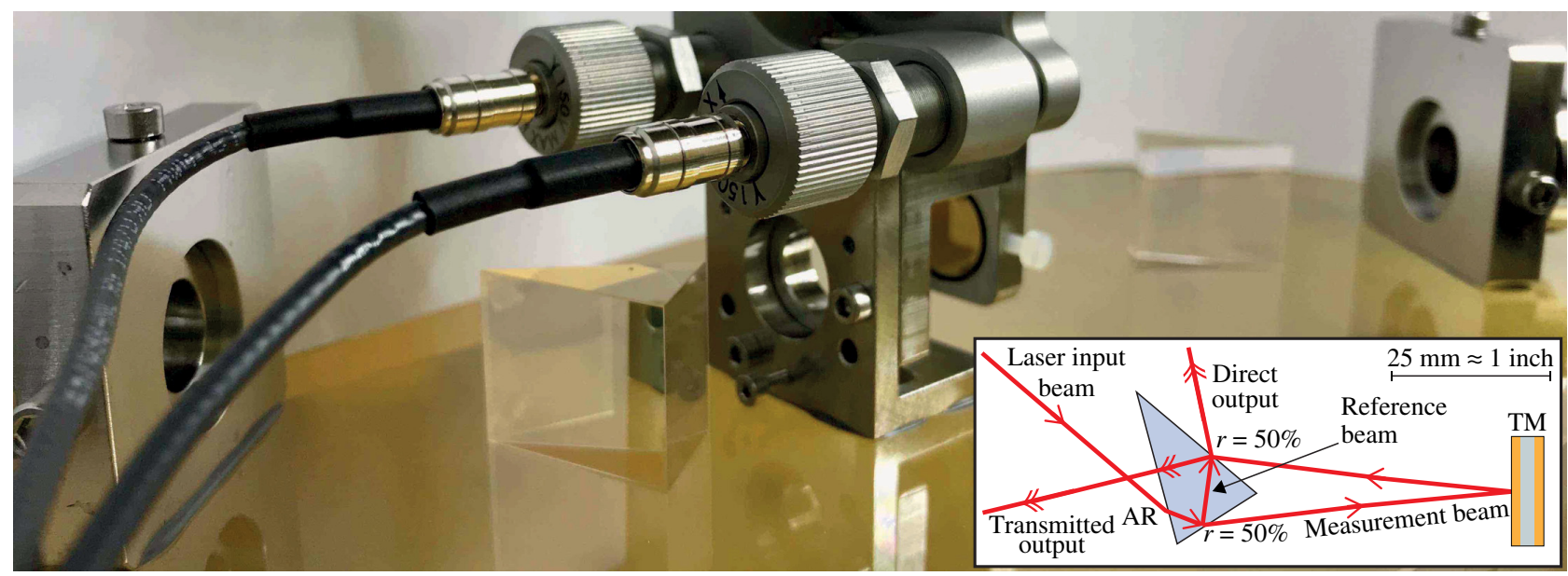

FIG. 2. A photograph of the TMitM. One prism is placed on each side of the gold-coated mirror (12.7 mm in diameter), which is suspended from a three-axis piezoactuator. Fiber collimators with Titanium housing are fixed in Invar holders to provide light to each interferometer. The inset shows a sketch of the left prism-shaped interferometer with its beam splitter and antireflective (AR) coating. The laser beam paths are drawn in red and hit the test mass (TM) under $4.1^{\circ}$.

reference beam travels a short distance inside the medium while the measurement beam leaves the medium and is reflected by the TM. The interferometer is designed for an angle of incidence of $4.1^{\circ}$ and an arm-length mismatch of $83.3 \mathrm{~mm}$, and it provides two redundant optical output ports, which we call the direct and transmitted ports. The transmitted port contains the interference signal with a phase shift of $\pi$ with respect to the direct one. The transmitted beams also leave the medium without compensating the previous refraction, which leads to beam-profile distortions in the transmitted port and an effective ellipticity of about 0.7 for both interfering beams.

Two prisms are glued with ultraviolet- (UV) cured adhesive onto the OB of the TMitM. Two adjustable fiber collimators provide the light for the prisms. Both interferometers are aligned such that after vacuum chamber evacuation we achieve maximum contrast (more than 95\%), taking into account a small alignment change due to the refractive index of air.

\section{Readout and control}

We count three interferometers inside the vacuum chamber that must be read out. Each interferometer has a slightly different modulation index due to its individual arm-length mismatch. The reference interferometer's modulation index is $m_{\mathrm{REF}}=7.5 \mathrm{rad}$ (stabilized). The two prism interferometers in the TMitM show an index of about $m_{L} \approx$ $8.9 \mathrm{rad}$ in the left one and $m_{R} \approx 9.1 \mathrm{rad}$ in the right one.

The readout algorithm of the DFMI is concisely illustrated in Fig. 1(c). The extraction of the desired fit parameters is done in real time in the phase meter and is implemented in a $\mathrm{C}$ program running on a personal computer (PC). An eight-channel data-acquisition card with a $250 \mathrm{kHz}$ sampling rate is used to digitize the photodiode voltages, which are generated from the photocurrents via transimpedance amplifiers. A digital-to-analog converter provides actuation signals from the phase meter via a universal serial bus (USB) interface. Five digital-feedback control loops (proportional-integral controllers) are integrated in the software, as follows. The tilt of the TM in the horizontal and vertical directions can be controlled by actuating on the three-axis PZT mount. As an error signal, we use a differential-wave-front-sensing (DWS) measurement, which is provided by quadrant photodiodes (QPDs). The limited number of readout channels allows us to only use one QPD during the displacement-performance measurements, which we use to actively suppress TM tilts. The coupling factor between the optical tilt and the DWS signal, of about $5000 \mathrm{rad} / \mathrm{rad}$, is calculated by means of optical simulations for an assumed beam waist radius of $0.5 \mathrm{~mm}$ and a waist position of $100 \mathrm{~mm}$; lenses with a 25.4-mm focal length produce a spot-size radius of 0.12 $\mathrm{mm}$ on the active area of the diodes. The experimental data verify this coupling factor. The path length of the TM is controlled by actuating on all three PZT axes simultaneously. The two remaining feedback loops control the average laser frequency and its applied FM deviation by tuning the modulation dc voltage and ac amplitude, respectively. The software phase meter restricts the bandwidths of all of the control loops to about $10 \mathrm{~Hz}$.

\section{RESULTS}

The TMitM measures two interferometric phases, $\varphi_{L}$ and $\varphi_{R}$, on the left and on the right side of the TM. The wave number $k=2 \pi / \lambda$ converts the phases into displacements, $\tilde{x}=\varphi / k$, and we can define $\tilde{x}_{L}$ and $\tilde{x}_{R}$ for the two sides and an equivalent $\tilde{x}_{\text {ref }}$ for the reference interferometer. A TM motion, $\Delta x$, is monitored redundantly in 
both prism interferometers except for an opposite sign. We introduce the parameter $\varepsilon$, which adds an additional noise term that is not common mode in the two interferometers. If one also includes the laser frequency noise, $v$, it couples into all interferometers scaled by their arm-length difference, given by $m_{L}, m_{R}$, and $m_{\text {ref }}$, as extracted by the phase meter. The displacement noise for each interferometer can be described as follows:

$$
\begin{aligned}
\tilde{x}_{L} & =+2 \Delta x+v m_{L}, \\
\tilde{x}_{R} & =-2 \Delta x+2 \varepsilon+v m_{R}, \\
\tilde{x}_{\text {ref }} & =\sigma+v m_{\text {ref. }}
\end{aligned}
$$

The test-mass displacement, $\Delta x$, occurs amplified by a factor of 2 in each prism measurement due to the reflection setup. With an angle of incidence of about $4.1^{\circ}$, this results in a coupling factor of nearly 2 , which is inserted in the above equations. The measurement of the reference interferometer contains an additional phase noise term, $\sigma$, that is driven by, e.g., wrong polarizations or other noise influences unique to this interferometer [23]. Equations (1)-(3) can be combined into two expressions:

$$
\begin{aligned}
\tilde{x}_{\mathrm{TM}} & :=\left(\tilde{x}_{L}-\tilde{x}_{R}\right) / 4 \approx \Delta x+\varepsilon / 2, \\
\tilde{x}_{\mathrm{RPN}} & :=\left(\tilde{x}_{L}+\tilde{x}_{R}-\tilde{x}_{\mathrm{ref}} \rho\right) / 4=\varepsilon / 2+\sigma \rho / 4,
\end{aligned}
$$

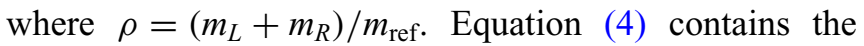
actual TM motion and the noise term $\varepsilon$. A minor coupling of residual frequency noise, $\mathcal{O}\left(m_{L}-m_{R}\right)$, is present but is found to be negligible here. Equation (5) combines all three measurements to reveal the total residual phase noise (RPN), containing the two noise terms $\sigma$ and $\varepsilon$. The RPN can be used to analyze any residual noise in the test bed or of the readout technique itself. A further signal combination is the optoelectronic noise (OEN), $\tilde{x}_{\mathrm{OEN}}$, which shows the difference between two redundant outputs of a recombination beam splitter, e.g., $\tilde{x}_{L \text {,direct }}-\tilde{x}_{L \text {,transmitted, which is }}$ a useful quantity for the evaluation of readout or stray light noise in the experiment.
The result of the TM displacement noise is plotted in Fig. 3. By applying all of the control schemes (laser frequency and/or modulation and TM stabilization), we are able to sense TM displacements with $230 \mathrm{fm} / \sqrt{\mathrm{Hz}}$ precision between $300 \mathrm{mHz}$ and $10 \mathrm{~Hz}$ ( $\left.\tilde{x}_{\mathrm{RPN}}\right)$. The TM motion, $\tilde{x}_{\mathrm{TM}}$, shows artifacts at higher harmonics that are induced by acoustic and seismic noise. The peak at $25 \mathrm{~Hz}$ is identified to be the oscillation of the vacuum pump running during this measurement. At frequencies below $30 \mathrm{mHz}$, the noise increases with a $1 / f^{2}$ behavior. The reasons for this are, to some extent, temperature fluctuations that cause beam jitter or other nonlinear effects.

\section{A. Nonlinearities}

Amplitude fluctuations couple into the optoelectronic noise, $\tilde{x}_{\mathrm{OEN}}$ and might be caused by the laser source itself or due to polarization fluctuations. The amplitude stabilization reduces this noise up to a bandwidth of $100 \mathrm{kHz}$. Digitization noise limits $\tilde{x}_{\mathrm{OEN}}$ at $150 \mathrm{fm} / \sqrt{\mathrm{Hz}}$ down to $30 \mathrm{mHz}$, as shown in Fig. 3. We assume that polarization fluctuations caused by refractive-index changes in the fiber components are the main reason for the increasing noise level below $30 \mathrm{mHz}$. A residual amplitude modulation caused by the applied strong frequency modulation and not sufficiently suppressed by the stabilization cannot be excluded either.

The reference interferometer is used as sensor to stabilize the laser frequency noise and to lock the modulation index to a constant value, here $m_{\text {ref }}=7.5 \mathrm{rad}$. The stabilization of the modulation index is useful to lock the operation point of the laser. While the control of the modulation index does not show any significant improvements in the phase performance, we are able to reduce laser frequency noise drifts below the unity gain frequency of $10 \mathrm{~Hz}$. By subtracting the remaining laser frequency noise in data postprocessing, we are able to further improve the performance between $100 \mathrm{mHz}$ and $50 \mathrm{~Hz}$ by one order of magnitude at $1 \mathrm{~Hz}$. The resulting residual phase noise is given by $\tilde{x}_{\text {RPN }}$ in Fig. 3 . It is limited by OEN above $1 \mathrm{~Hz}$ and by some unexplained noncommon mode noise

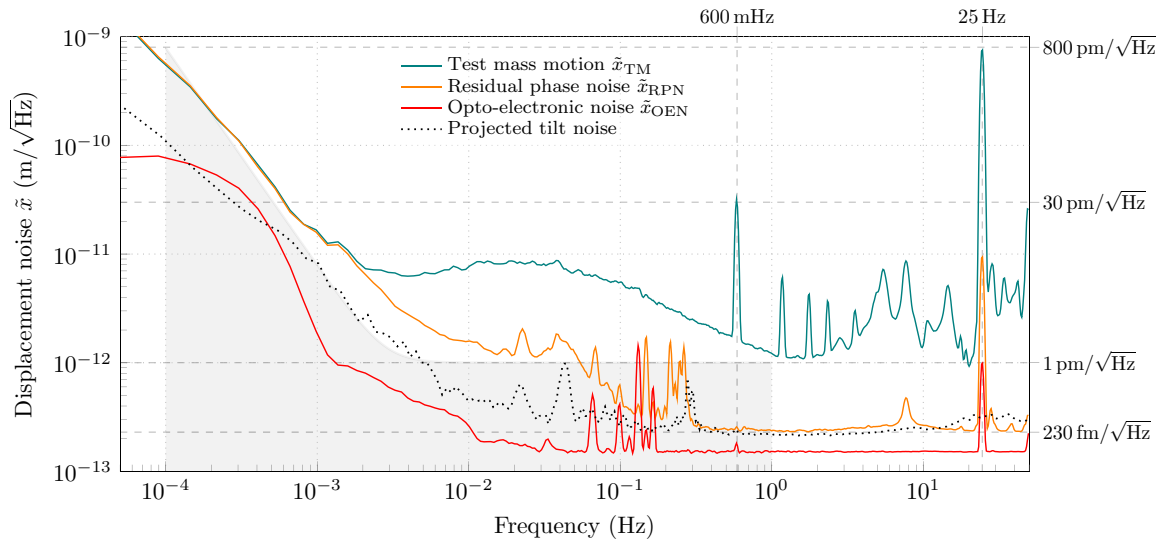

FIG. 3. The displacement performance of DFMI. As a reference, we plot the sensitivity curve of the LISA missionconcept document of $1 \mathrm{pm} / \sqrt{\mathrm{Hz}}$ relaxed to frequencies below $3 \mathrm{mHz}$ as the gray area [4]. The individual signals are calculated from the interferometric phases according to Eqs. (4) and (5). Shot noise is not visible in this plot and is expected to be below $10^{-14} \mathrm{~m} / \sqrt{\mathrm{Hz}}$. 
source below this frequency. Despite this excess noise, the measurement shows that DFMI with single-component interferometers is able to achieve a 1-pm-level precision comparable to, and partly better than, previous breadboard experiments for LISA and LISA pathfinder [7,25-27].

The measurement also shows a dynamic range of more than 2 orders of magnitude at $600 \mathrm{mHz}$ and $25 \mathrm{~Hz}$ between $\tilde{x}_{\mathrm{TM}}$ and $\tilde{x}_{\mathrm{RPN}}$. This is compliant with gravity missions that use some form of feedback control, such as LISA, LISA Pathfinder, or gravity-field and steadystate ocean-circulation explorer (GOCE) type instruments. Residual readout nonlinearities might be caused by the strong frequency modulation itself that could limit the dynamic range capabilities, as discussed in previous studies $[11,13]$. Possible reasons are harmonic distortions of the modulation caused by the function generator or the laser itself via the external-cavity excitation. In the current range, these effects do not seem to be limiting; however, they might become dominant for higher dynamics if no further compensation is applied. The influence of nonflat transfer functions of the photoreceivers is also measured and corrected and is most probably not the limiting factor at this point. Other geometrical effects, such as nonparallel incidence or a wedge of the TM, can also lead to nonlinear coupling. The current noise limitations are, however, more likely explained by undesired tilts, as described in the following.

\section{B. Tilt-to-length noise}

In a subsequent measurement, we use a second QPD in the other prism interferometer and monitor the out-ofloop behavior of the TM tilts. Due to the limited number of readout channels, we are not able to stabilize the laser frequency and modulation or the TM path length during this additional measurement. Only the TM tilt noise is monitored and the corresponding in- and out-of-loop measurements are shown in Fig. 4. We are able to stabilize the TM tilt down to noise levels on the order of $20 \mathrm{nrad} / \sqrt{\mathrm{Hz}}$ above $40 \mathrm{mHz}$, as the out-of-loop measurements indicate. The projected TM displacement noise can be determined by combining the in-loop and out-of-loop tilt measurements. The DWS and tilt-to-length coupling factors for beam jitter are determined by optical simulations and are about $5000 \mathrm{rad} / \mathrm{rad}$ and $4 \mathrm{pm} / \mu \mathrm{rad}$, including some initial interferometric misalignment of about $50 \mu \mathrm{rad}$. The projected tilt noise is shown by the dotted black line plotted in Fig. 3. It matches the residual phase noise, $\tilde{x}_{\text {RPN }}$, above $200 \mathrm{mHz}$ and might explain some of the noise increase below $200 \mathrm{mHz}$. Not measured here are the beam-pointing fluctuations of the reference interferometer, which likely also drive the performance of $\tilde{x}_{\text {RPN }}$ by coupling into the measurement via the residual noise of the laser frequency stabilization [23]. This could explain the missing correlation between the projected tilt noise and the residual phase noise measurement at low frequencies.

\section{SUMMARY}

The use of DFMI allows simpler interferometer setups in comparison to heterodyne interferometry. In particular, it scales more easily to multiple TM displacement and tilt readouts on the same or adjacent ultrastable benches. It can further maintain multifringe capabilities that are required, for example, for a TM readout in space. Only one frequency reference interferometer is required per laser source. The thus prepared laser light can be distributed into many optical setups, the number of which is eventually constrained by the minimal optical power required not to be limited by shot noise.

Using a compact single-component beam splitting and recombination optic specifically designed for DFMI, we conduct a prototype test-mass experiment in which we are able to reach displacement-sensing levels of $230 \mathrm{fm} / \sqrt{\mathrm{Hz}}$ around $300 \mathrm{mHz}$ and tilt-noise readouts with sensitivities of about $20 \mathrm{nrad} / \sqrt{\mathrm{Hz}}$ at $40 \mathrm{mHz}$. The current performance limitation at low frequencies is most probably caused by beam-pointing fluctuations, which can be reduced in future experiments by the use of ultrastable monolithic fiber collimators. At high frequencies, we are

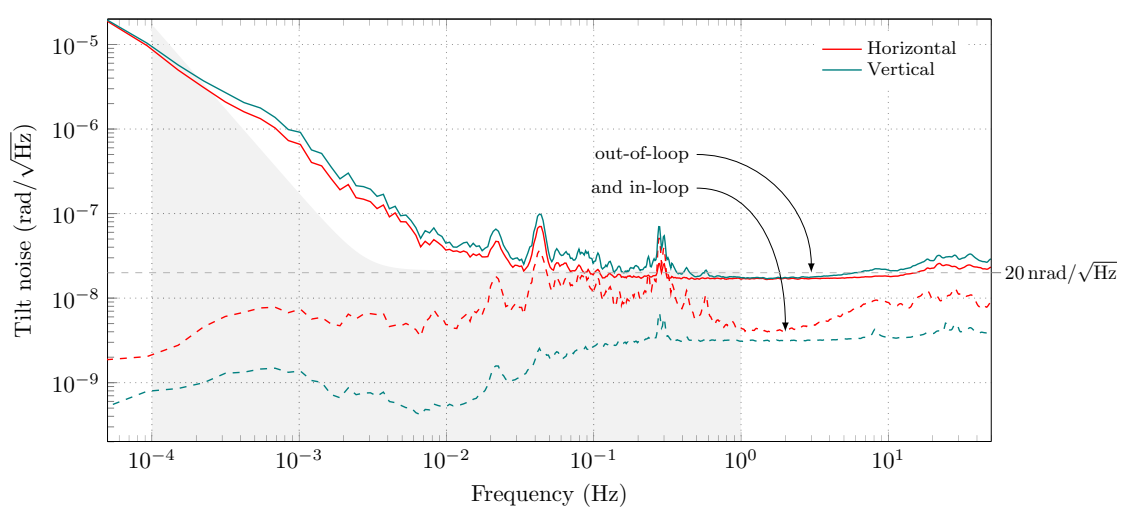

FIG. 4. The tilt-noise performance of DFMI. As a reference, we plot the mission requirements of the LPF test campaign of $20 \mathrm{nrad} / \sqrt{\mathrm{Hz}}$ relaxed to frequencies below $3 \mathrm{mHz}$ as the gray area [28]. To get the optical tilt-noise performance, we divide the measured DWS phase measurement by the coupling factor (approximately 5000 $\mathrm{rad} / \mathrm{rad})$. 
currently limited by the digitization noise of our dataacquisition system. The required strong frequency modulation causes nonlinearities that are discussed and found not to be limiting in the dynamic range regime probed here. Future work will concentrate on the development of scalable phase meters with many channels and on optimizing the readout and laser-control algorithms to further test and increase the phase-readout linearity. The experiment represented here is a test bed that is well suited to these future investigations.

To conclude, the simplicity of the compact interferometric setup and the achieved LISA-like performance make DFMI attractive for future experiments and metrology applications.

\section{ACKNOWLEDGMENTS}

The authors would like to thank the Deutsche Forschungsgemeinschaft (DFG) Sonderforschungsbereich 1128 Relativistic Geodesy and Gravimetry with QuantumSensors (geo-Q) and the International Max-Planck Research School (IMPRS) for financial support.

[1] M. Armano, H. Audley, J. Baird, P. Binetruy, M. Born, D. Bortoluzzi, E. Castelli, A. Cavalleri, A. Cesarini, A. M. Cruise et al., Beyond the Required LISA Free-fall Performance: New LISA Pathfinder Results Down to $20 \mathrm{~Hz}$, Phys. Rev. Lett. 120, 061101 (2018).

[2] M. R. Drinkwater, R. Haagmans, D. Muzi, A. Popescu, R. Floberghagen, M. Kern, and M. Fehringer, in Proceedings of the 3rd International GOCE User Workshop (European Space Agency Noordwijk, The Netherlands, 2006), p. 6.

[3] S. Cooper, C. Collins, A. Green, D. Hoyland, C. Speake, A. Freise, and C. Mow-Lowry, A compact, large-range interferometer for precision measurement and inertial sensing, Class. Quantum Gravity 35, 095007 (2018).

[4] P. Amaro-Seoane, H. Audley, S. Babak, J. Baker, E. Barausse, P. Bender, E. Berti, P. Binetruy, M. Born, D. Bortoluzzi et al., Laser interferometer space antenna, arXiv:1702.00786.

[5] K. Dahl, G. Heinzel, B. Willke, K. A. Strain, S. Goler, and K. Danzmann, Suspension platform interferometer for the AEI 10 m prototype: Concept, design and optical layout, Class. Quantum Gravity 29, 095024 (2012).

[6] B. P. Abbott, R. Abbott, T. D. Abbott, F. Acernese, K. Ackley, C. Adams, T. Adams, P. Addesso, R. X. Adhikari, V. B. Adya et al., GW170814: A Three-detector Observation of Gravitational Waves from a Binary Black Hole Coalescence, Phys. Rev. Lett. 119, 141101 (2017).

[7] J. Watchi, S. Cooper, B. Ding, C. M. Mow-Lowry, and C. Collette, Contributed review: A review of compact interferometers, Rev. Sci. Instrum. 89, 121501 (2018).

[8] A. J. Sutton, O. Gerberding, G. Heinzel, and D. A. Shaddock, Digitally enhanced homodyne interferometry, Opt. Express 20, 22195 (2012).
[9] T. S. Schwarze, O. Gerberding, F. G. Cervantes, G. Heinzel, and K. Danzmann, Advanced phasemeter for deep phase modulation interferometry, Opt. Express 22, 18214 (2014).

[10] G. Heinzel, F. Guzmán Cervantes, A. F. Garcia Marin, J. Kullmann, W. Feng, and K. Danzmann, Deep phase modulation interferometry, Opt. Express 18, 19076 (2010).

[11] O. Gerberding, Deep frequency modulation interferometry, Opt. Express 23, 14753 (2015).

[12] K.-S. Isleif, O. Gerberding, M. Mehmet, T. S. Schwarze, G. Heinzel, and K. Danzmann, Comparing interferometry techniques for multi-degree of freedom test mass readout, J. Phys. Conf. Ser. 716, 012008 (2016).

[13] K.-S. Isleif, O. Gerberding, T. S. Schwarze, M. Mehmet, G. Heinzel, and F. G. Cervantes, Experimental demonstration of deep frequency modulation interferometry, Opt. Express 24, 1676 (2016).

[14] D. A. Shaddock, Digitally enhanced heterodyne interferometry, Opt. Lett. 32, 3355 (2007).

[15] K.-S. Isleif, O. Gerberding, S. Köhlenbeck, A. Sutton, B. Sheard, S. Goßler, D. Shaddock, G. Heinzel, and K. Danzmann, Highspeed multiplexed heterodyne interferometry, Opt. Express 22, 24689 (2014).

[16] T. Kissinger, T. O. H. Charrett, and R. P. Tatam, Fibre segment interferometry using code-division multiplexed optical signal processing for strain sensing applications, Meas. Sci. Technol. 24, 094011 (2013).

[17] T. Kissinger, T. O. Charrett, and R. P. Tatam, Rangeresolved interferometric signal processing using sinusoidal optical frequency modulation, Opt. Express 23, 9415 (2015).

[18] G. De Vine, D. S. Rabeling, B. J. Slagmolen, T. T. Lam, S. Chua, D. M. Wuchenich, D. E. McClelland, and D. A. Shaddock, Picometer level displacement metrology with digitally enhanced heterodyne interferometry, Opt. Express 17, 828 (2009).

[19] S. A. Akbarzadeh-Jahromi and M. Shahabadi, Low-cost highly sensitive refractive index measurement based on deep frequency modulation interferometry, IEEE Sens. J. 17, 5460 (2017).

[20] C. Ni, M. Zhang, Y. Zhu, C. Hu, S. Ding, and Z. Jia, Sinusoidal phase-modulating interferometer with ellipse fitting and a correction method, Appl. Opt. 56, 3895 (2017).

[21] M. Zhang, C. Ni, Y. Zhu, L. Wang, C. Hu, and J. Hu, Largerange displacement measurement using sinusoidal phasemodulating laser diode interferometer, Chin. Opt. Lett. 15, 101201 (2017).

[22] M. Arablu and S. T. Smith, Polydyne displacement interferometer using frequency-modulated light, Rev. Sci. Instrum. 89, 055007 (2018).

[23] O. Gerberding, K.-S. Isleif, M. Mehmet, K. Danzmann, and G. Heinzel, Laser-frequency Stabilization via a Quasimonolithic Mach-Zehnder Interferometer with Arms of Unequal Length and Balanced dc Readout, Phys. Rev. Appl. 7, 024027 (2017).

[24] G. Wanner, G. Heinzel, E. Kochkina, C. Mahrdt, B. S. Sheard, S. Schuster, and K. Danzmann, Methods for simulating the readout of lengths and angles in laser interferometers with Gaussian beams, Opt. Commun. 285, 4831 (2012). 
[25] G. Heinzel, V. Wand, A. Garcia, O. Jennrich, C. Braxmaier, D. Robertson, K. Middleton, D. Hoyland, A. Rüdiger, R. Schilling, U. Johann, and K. Danzmann, The LTP interferometer and phasemeter, Class. Quantum Gravity 21, S581 (2004).

[26] G. de Vine, B. Ware, K. McKenzie, R. E. Spero, W. M. Klipstein, and D. A. Shaddock, Experimental Demonstration of Time-Delay Interferometry for the Laser Interferometer Space Antenna, Phys. Rev. Lett. 104, 211103 (2010).
[27] T. Schuldt, M. Gohlke, H. Kögel, R. Spannagel, A. Peters, U. Johann, D. Weise, and C. Braxmaier, Picometre and nanoradian heterodyne interferometry and its application in dilatometry and surface metrology, Meas. Sci. Technol. 23, 054008 (2012).

[28] F. G. Cervantes, R. Flatscher, D. Gerardi, J. Burkhardt, R. Gerndt, M. Nofrarias, J. Reiche, G. Heinzel, K. Danzmann, L. G. Boté, V. Martí, I. Mateos, and A. Lobo, LISA technology package flight hardware test campaign, APS Conf. Ser. (2013), Vol. 467, p. 141. 